\title{
Nonlinear Aharonov-Bohm Scattering by Optical Vortices
}

\author{
Dragomir Neshev, ${ }^{1}$ Alexander Nepomnyashchy, ${ }^{1,2}$ and Yuri S. Kivshar ${ }^{1}$ \\ ${ }^{1}$ Nonlinear Physics Group, Research School of Physical Sciences and Engineering, The Australian National University, \\ Canberra, ACT 0200, Australia \\ ${ }^{2}$ Department of Mathematics and Minerva Center for Nonlinear Physics of Complex Systems, \\ Technion-Israel Institute of Technology, 32000 Haifa, Israel \\ (Received 28 December 2000; published 3 July 2001)
}

\begin{abstract}
We study linear and nonlinear wave scattering by an optical vortex in a self-defocusing nonlinear Kerr medium. In the linear case, we find a splitting of a plane-wave front at the vortex proportional to its circulation, similar to what occurs in the scattered wave of electrons for the Aharonov-Bohm effect. For larger wave amplitudes, we study analytically and numerically the scattering of a dark-soliton stripe (a nonlinear analog of a small-amplitude wave packet) by a vortex and observe a significant asymmetry of the scattered wave. Subsequently, a wave-front splitting of the scattered wave develops into transverse modulational instability, "unzipping" the stripe into trains of vortices with opposite charges.
\end{abstract}

DOI: $10.1103 /$ PhysRevLett.87.043901

PACS numbers: $42.65 . \mathrm{Tg}, 03.75 . \mathrm{Fi}$

It is known that the magnetic vector potential influences the dynamics of a charged quantum particle even if the magnetic field vanishes, e.g., when the field is confined to a cylinder into which electrons cannot penetrate. This phenomenon is known these days as the Aharonov-Bohm effect [1], and it was shown to have a more general geometrical framework being directly linked to the wave-front dislocations and geometrical phases [2]. It was also shown to have a classical analog in the scattering of a linear wave by a vortex [3]. Recently, the study of the interaction of a water wave with a vortex [4] revealed both similarities and differences with the Aharonov-Bohm effect, allowing one to observe directly the macroscopic aspects of the geometrical phases.

In this Letter we study an optical analog of the Aharonov-Bohm effect - the wave scattering by an optical vortex in a bulk dielectric nonlinear medium. We describe, for the first time to our knowledge, nonlinear effects in the Aharonov-Bohm scattering including both the crossover regime and the strongly nonlinear case. Since our basic equations describe also the dynamics of a superfluid flow in the Bose-Einstein condensates [5], we expect that our results will be useful for the experimental study of the similar effects in the nonlinear matter-wave physics.

Model.-We consider the propagation of a monochromatic scalar electric field in a bulk nonlinear Kerr-like medium with an intensity-dependent refractive index, $n=$ $n_{0}+n_{2} I$, where $n_{2}$ is the Kerr-effect coefficient characterizing a defocusing nonlinearity $\left(n_{2}<0\right)$. In the paraxial approximation, the Maxwell equations can be reduced to the nonlinear Schrödinger (NLS) equation for the renormalized slowly varying amplitude $\psi$,

$$
i \frac{\partial \psi}{\partial z}+\frac{1}{2} \nabla_{\perp}^{2} \psi+\left(1-|\psi|^{2}\right) \psi=0,
$$

where the propagation coordinate $(z)$ and the transverse coordinates $(x, y)$ are measured in the units of $k_{0}\left|n_{2}\right| I_{0}$ and $\left(n_{0}\left|n_{2}\right| k_{0}^{2} I_{0}\right)^{1 / 2}$, respectively. Here $k_{0}$ is the free-space wave number, $n_{0}$ is the linear refractive index, $\nabla_{\perp}$ is the transverse gradient operator, and $I=|E|^{2}$ is the field intensity (see details in Ref. [6]).

Equation (1) has a stationary solution for a vortex [6], $\psi_{0}(r)=R_{0}(r) e^{i \varphi}$, where the real amplitude $R_{0}(r)$ satisfies the conditions $R_{0}(0)=0$, and $R_{0}(r)=1+O\left(1 / r^{2}\right)$, as $r \rightarrow \infty$. To study the wave scattering by the vortex, we introduce a new function $\Phi(r, z)$, substituting $\psi(r, z)=$ $\psi_{0}(r) \Phi(r, z)$ into Eq. (1) and taking into account the lowest-order terms in $r^{-1}$. The corresponding equation for $\Phi(r, z)$ has the form

$$
\begin{aligned}
i\left(\frac{\partial \Phi}{\partial z}+\frac{\left(\vec{e}_{\varphi} \cdot \nabla_{\perp}\right) \Phi}{r}\right)+ & \frac{1}{2} \nabla_{\perp}^{2} \Phi+ \\
& \left(1-|\Phi|^{2}\right) \Phi=0,
\end{aligned}
$$

and it describes the advection of the field $\Phi(r, z)$ by the vortex phase gradient field $\vec{e}_{\varphi} / r$.

Linear limit: the Aharonov-Bohm scattering.-First, we analyze the scattering of a small-amplitude wave on a vortex. This problem was studied earlier in the context of the superfluid hydrodynamics [7]. Herewith, we develop a different approach that allows a simple further extension to the nonlinear regime.

Let us consider a plane-wave front with the coordinate $x=X(y)$ that moves in the $x$ direction with the velocity $v$ [see Fig. 1(a)] and is advected simultaneously by the vortex velocity field $u^{(0)}(y, z)=-\left(y / r^{2}\right)=-y /\left(y^{2}+\right.$ $\left.v^{2} z^{2}\right)$. If the wave-front motion starts at $z=z_{\text {in }}<0$ and the point $z=0$ corresponds to the moment when the unperturbed line crosses the vortex [see Fig. 1(c)], the shape of the line in the $y$ direction, $h^{(0)}(y, z)$, can be found as (for $|y| \gg 1$ )

$$
h^{(0)}(y, z)=-\frac{\operatorname{sgn}(y)}{v}\left(\tan ^{-1} \frac{v z}{|y|}-\tan ^{-1} \frac{v z_{\text {in }}}{|y|}\right) .
$$

Thus, the total deformation of the wave front can be 

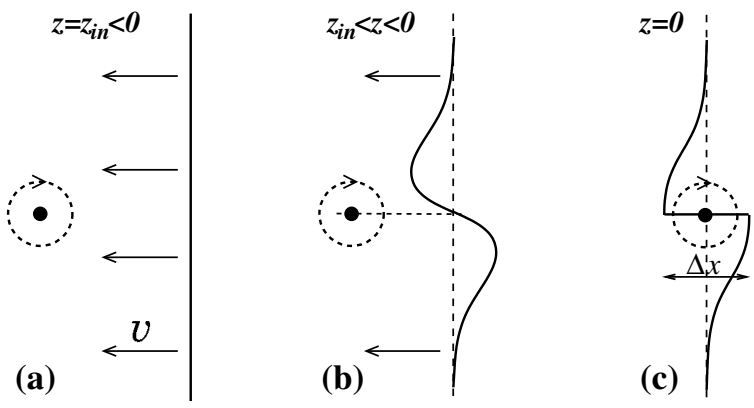

FIG. 1. Sketch of the wave-front deformation and splitting at different distances from the vortex.

calculated as follows (for $v|z|, v\left|z_{\text {in }}\right| \gg|y| \gg 1$ ):

$$
h^{(0)}(y, z)=-\frac{\operatorname{sgn}(y)}{v} \pi .
$$

Equation (3) coincides with the result given by Eq. (4.73) of Ref. [7] provided $v$ has a meaning of the sound speed. It describes a splitting of the wave front by the amount $\Delta x=-2 \pi / v$, predicted by the Aharonov-Bohm effect for the linear waves scattered by a vortex.

Nonlinear regime: Deformation of a dark-soliton stripe. - In the nonlinear regime of the vortex-wave scattering, the steady-state propagation of the wave packet

$$
\frac{1}{2} \frac{\partial^{2} \Phi^{(1)}}{\partial \xi^{2}}-i v \frac{\partial \Phi^{(1)}}{\partial \xi}+\left(1-2\left|\Phi^{(0)}\right|^{2}\right) \Phi^{(1)}-\Phi^{(0) 2} \Phi^{*(1)}=i V(Y, Z) \frac{\partial \Phi^{(0)}}{\partial \xi},
$$

where

$$
V(Y, Z)=\frac{\partial h}{\partial Z}+\frac{Y}{Y^{2}+v^{2} Z^{2}} .
$$

A solution of Eq. (5) can be found as $\Phi^{(1)}=V(Y, Z) \times$ $\left(\partial \Phi^{(0)} / \partial v\right)$. Result (6), being expressed as $h_{Z}=V-$ $Y\left(Y^{2}+v^{2} Z^{2}\right)^{-1}$, has a simple physical meaning: a local change of the stripe coordinate, $h_{Z}$, is a sum of its local velocity, $V$, and a correction produced by the vortex.

In the second order in $\epsilon$, we obtain an equation for $\Phi^{(2)}$ similar to Eq. (5) with different right-hand side (rhs) terms. The equation governing the evolution of the dark-solitonstripe parameters can be obtained from the orthogonality condition of the rhs of the equation for $\Phi^{(1)}$ to the solution of the homogeneous problem, $\Phi^{(1)}=\partial \Phi^{(0)} / \partial \xi$. Taking into account the parity properties and assuming the normalization $\operatorname{Im}\left(\partial \Phi^{(0)} / \partial v\right)=1$, we derive the equation

$$
\frac{\partial V}{\partial Z}+\lambda^{2}(v) \frac{\partial^{2} h}{\partial Y^{2}}=0,
$$

where $\lambda^{2}(v)=\frac{1}{3}\left(1-v^{2}\right)$. The system of Eqs. (6),(7) describes the evolution of the stripe parameters in the presence of a vortex field, and it can be rewritten in the original variables $(y, z)$, if we define $\tilde{u}=\epsilon V$, and $h_{Z}=\tilde{u}+u^{(0)}$, as follows:

$$
\frac{\partial^{2} \tilde{u}}{\partial z^{2}}+\lambda^{2} \frac{\partial^{2} \tilde{u}}{\partial y^{2}}=-\lambda^{2} \frac{\partial^{2} u^{(0)}}{\partial y^{2}}
$$

in the presence of nonlinearity is possible in the form of a solitary wave when nonlinear effects are balanced by the wave dispersion [8]. In the problem under consideration, such a solitary wave corresponds to a localized intensity dip on a constant plane-wave background, a spatial darksoliton stripe [6]. The corresponding nonlinear scattering problem is rather complicated, and we restrict our study by the region of large distances from the vortex core, $r=O\left(\epsilon^{-1}\right)$, where $\epsilon \ll 1$ is a formal small parameter of the asymptotic analysis we develop below.

We assume that, under the action of the vortex field, a dark-soliton stripe moves with the velocity $v=O(1)$ and the coordinate of its center gets shifted, in respect to its unperturbed motion $x=v z$, by the amount $h(\epsilon y, \epsilon z)=$ $O(1)$. Introducing new variables $Y=\epsilon y, Z=\epsilon z, \xi=$ $x-v z-h(Y, Z)$, we look for the solution in the asymptotic form, $\Phi=\sum_{n=0}^{\infty} \epsilon^{n} \Phi^{(n)}$. In the zero order in $\epsilon$, we find

$$
\frac{1}{2} \frac{\partial^{2} \Phi^{(0)}}{\partial \xi^{2}}-i v \frac{\partial \Phi^{(0)}}{\partial \xi}+\left(1-\left|\Phi^{(0)}\right|^{2}\right) \Phi^{(0)}=0,
$$

that has a well-known solution for a dark soliton [6]

$$
\Phi^{(0)}=\left[\sqrt{1-v^{2}} \tanh \left(\sqrt{1-v^{2}} \xi\right)+i v\right] e^{i \theta} .
$$

In the first order in $\epsilon$, we obtain the equation

Nonlinear wave-front splitting.-The Cauchy problem for the elliptic equation (8) is ill-posed; hence its solutions are meaningful only if the generation of short-wavelength harmonics is not essential. To solve Eq. (8), first we apply

$$
\frac{d^{2} u_{k}}{d z^{2}}-k^{2} \lambda^{2} u_{k}=i \pi k^{2} \lambda^{2} \operatorname{sgn}(k) e^{-|k| v|z|},
$$

where $u_{k}(z)$ is the Fourier transform of the field $\tilde{u}(y, z)$. Except the special case $\lambda=v$, the solution of Eq. (9) can be found in the general form

$$
u_{k}= \begin{cases}A_{k}^{-} e^{k \lambda z}+B_{k}^{-} e^{-k \lambda z}+a_{k} e^{|k| v z}, & z<0, \\ A_{k}^{+} e^{k \lambda z}+B_{k}^{+} e^{-k \lambda z}+a_{k} e^{-|k| v z}, & z>0,\end{cases}
$$

where $a_{k}=i \pi \lambda^{2}\left(v^{2}-\lambda^{2}\right)^{-1} \operatorname{sgn}(k)$, and the values of the amplitudes $A_{k}^{ \pm}$and $B_{k}^{ \pm}$are determined from the continuity conditions,

$$
A_{k}^{+}-A_{k}^{-}=-\left(B_{k}^{+}-B_{k}^{-}\right)=\frac{i \pi \lambda v}{\left(v^{2}-\lambda^{2}\right)} .
$$

First, we consider the region $z<0$. If the stripe instability is weak, i.e., $\lambda<v$, the third term in Eq. (10) dominates, and we find the result: $\tilde{u}(y, z) \approx\left[\lambda^{2} /\left(v^{2}-\right.\right.$ $\left.\left.\lambda^{2}\right)\right] u^{(0)}(y, z)$. Thus, the deformation of a dark-soliton stripe due to its interaction with the vortex field can be the Fourier transformation and obtain the equation 
presented in the form

$$
h(y, z)=\frac{v^{2}}{\left(v^{2}-\lambda^{2}\right)} h^{(0)}(y, z), \quad z<0,
$$

where $h^{(0)}(y, z)$ is defined by Eq. (2) of the linear theory. Therefore, the result (11) describes the so-called nonlinear Aharonov-Bohm scattering on the vortex; it is stronger than its linear analog by the factor $v^{2} /\left(v^{2}-\lambda^{2}\right)$, provided $\lambda<v$ as $v>1 / 2$.

The general solution (10) describes the growth of instability of a dark-soliton stripe. If this instability is rather strong (i.e., $\lambda>v$ ), the term with $A_{k}^{-}$becomes important, and the amplitude coefficients $A_{k}^{-}$and $B_{k}^{-}$should be determined from the initial conditions at $z=z_{\text {in }}$. If $h\left(y, z_{\text {in }}\right)=h_{z}\left(y, z_{\text {in }}\right)=0$, in the presence of a vortex the function $u_{k}$ grows linearly, for small $z+z_{\text {in }}$, and exponentially, for large $z+z_{\text {in }}$. This type of the stripe evolution was discussed earlier in Ref. [9] for the special case $v=0$

More importantly, we can find an exact nonsingular analytical solution of Eq. (10) for any $v$ and $\lambda$. Assuming $z=z_{\text {in }}<0$ and selecting the initial conditions in the form $\tilde{u}\left(y, z_{\text {in }}\right)=\tilde{u}_{z}\left(y, z_{\text {in }}\right)=0, \quad h\left(y, z_{\text {in }}\right)=0, \quad h_{z}\left(y, z_{\text {in }}\right)=$ $u^{(0)}\left(y, z_{\text {in }}\right)$, we solve Eq. (9) and find the dark-soliton deformation as follows:

$$
\begin{aligned}
h(y, z)=[ & \frac{1}{2(v+\lambda)} \tan ^{-1}\left(\frac{-\lambda z+(v+\lambda) z_{\text {in }}}{|y|}\right) \\
& +\frac{1}{2(v-\lambda)} \tan ^{-1}\left(\frac{\lambda z+(v-\lambda) z_{\text {in }}}{|y|}\right) \\
& \left.-\frac{v}{v^{2}-\lambda^{2}} \tan ^{-1}\left(\frac{v z}{|y|}\right)\right] \operatorname{sgn}(y) .
\end{aligned}
$$

The exact analytical solution (12) is more general than the result (11), and it transforms into Eq. (11) for $\mid(v-$ $\lambda) z_{\text {in }} / y \mid \gg 1$. Solution (12) remains valid even for $v \rightarrow$ 0 , i.e., for deformations of a black soliton.

In the region $z>0$, the exponentially growing term $\sim A_{k}^{+}$of Eq. (10) dominates, and the corresponding divergence at large $k$ is a direct manifestation of the ill-posed problem (8). It can be regularized by the higher-order term $\sim \beta h_{z y y}$, which appears in the equation $h_{z z}+\lambda^{2} h_{y y}=$ $u_{z}^{(0)}$ due to the effect of radiation taken into account in the asymptotic expansion (see details of such a technique in Ref. [10]). This term makes the linear problem (8) well posed, but it appears with additional nonlinear corrections that all describe the dynamics of the transverse instability of the dark-soliton stripe in the presence of a vortex field. The asymptotic analysis becomes very complicated and, in order to analyze the long-term dynamics of the nonlinear wave scattering, below we carry out numerical simulations.

Numerical simulations have been conducted by means of a standard beam propagation method, solving Eq. (1) over a numerical grid of $1024 \times 1024$ points. As initial conditions in the nonlinear problem, we use a superposition of a vortex and a dark-soliton stripe: $\psi(x, y, 0)=$ $\psi_{0}(r) \Phi(x, 0)$, where $\psi_{0}(r)=R_{0}(r) e^{i \varphi}$ and $R_{0}(r)$ describes the stationary solution for an optical vortex soliton found numerically by a shooting technique. The function $\Phi(x, 0)$ describes a dark-soliton stripe with an initial offset from the position of the vortex $\left(x_{0}\right)$, and it is given by Eq. (4). To ensure unperturbed propagation of an optical beam over long distances, the initial function $\psi(x, y, 0)$ was superimposed on a super-Gaussian-beam background with HWHM more than 40 times larger than the characteristic scale of the localized structures and interaction domain.

In our simulations, we vary the contrast of the darksoliton stripe (i.e., its transverse velocity) and monitor the stripe deformation for different propagation distances. First of all, we investigate the scattering of a smallamplitude dark-soliton stripe by an optical vortex, which should correspond to the scattering of a linear wave packet associated with the Aharonov-Bohm effect. In Fig. 2(a), we show the snapshots of the scattering process for the dark-soliton stripe with the transverse velocity $v^{2}=0.9$, at different propagation distances $z$ (marked on the figure). Below in Figs. 2(b) and 2(c), we show the details of the stripe deformation (first symmetric but then asymmetric) during the scattering, and the vortex shift $\Delta$ induced by the stripe scattering [11].

To investigate the strong nonlinear regime of the vortexstripe scattering we consider different values of the stripe amplitude by changing its velocity and, therefore, contrast. The results of these calculations are summarized in Fig. 3, where we show the results of the scattering process at the propagation distance $z=16$, similar to the corresponding plot in Fig. 2(a). We notice that the transverse

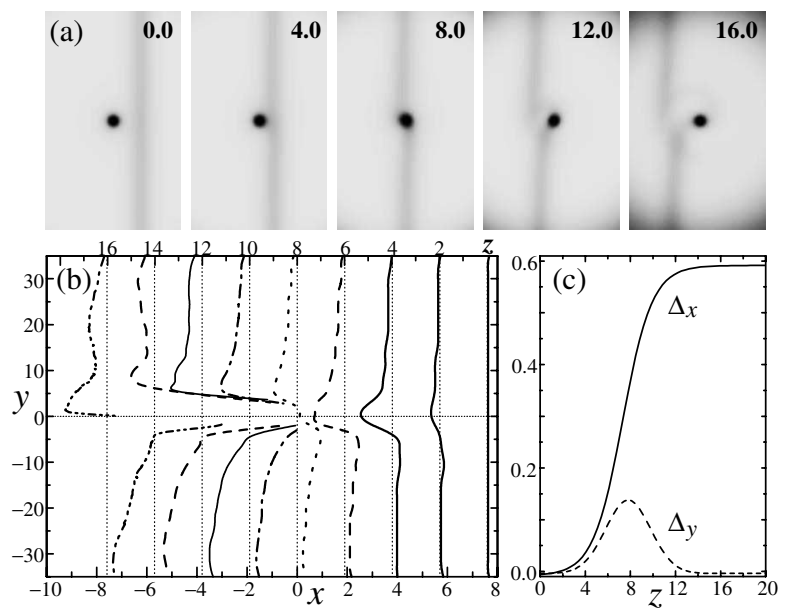

FIG. 2. (a) Snapshots of the interaction of an optical vortex with a dark-soliton stripe $\left(v^{2}=0.9\right)$. Initially, the stripe is shifted at $x_{0}=7.59$ from the vortex and, if propagating alone, it reaches the origin at $z=8$. For better visualization, $16 \%$ of the computational domain is shown, and the contrast of the images was adjusted. (b) Asymmetric stripe deformation at different propagation distances. (c) Vortex horizontal $\left(\Delta_{x}\right)$ and vertical $\left(\Delta_{y}\right)$ shift from its initial position. 


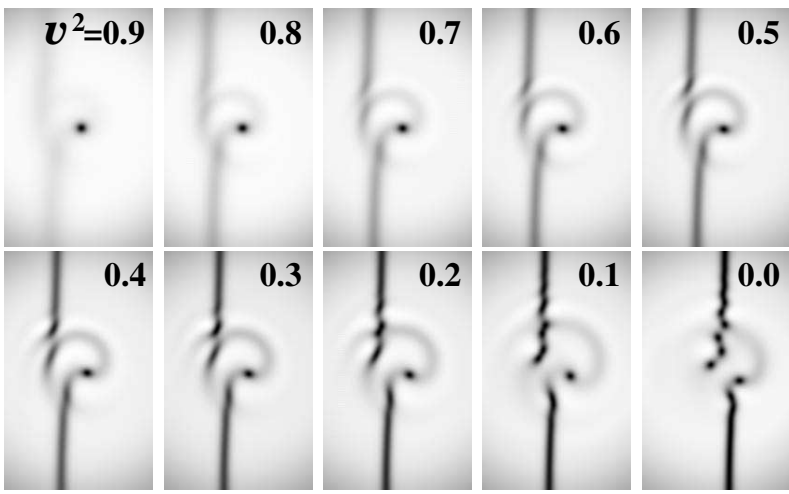

FIG. 3. Snapshots of a dark-soliton stripe scattered by an optical vortex soliton, after propagation $z=16$ and at different initial velocities $v^{2}$. The initial offset of the stripe is set such that if alone the stripe reaches the origin at $z=8$, except for the case $v=0$ where $x_{0}=2.5$.

modulational instability of the stripe starts developing for intermediate dark-stripe contrasts, and it is accompanied by a subsequent formation of mixed (edge-screw) and, later, screw phase dislocations. Consequently, the stripe breaks up into vortices of opposite charges in a way that resembles the dynamics and formation of vortex streets in the hydrodynamics. This phenomenon can be understood as an effective "unzipping" of the dark-soliton stripe by the vortex, that is, a manifestation of a strongly nonlinear regime in the Aharonov-Bohm scattering, a remarkable nonlinear effect.

Additionally, we notice that the primary vortex undergoes a large shift from its initial position after the scattering. In order to understand this effect, we have studied this process in detail (see Fig. 4) and have observed a novel type of interaction associated with the creation/ annihilation process of a vortex pair. Indeed, during the interaction, a part of the stripe closest to the vortex breaks up creating a pair of vortices with opposite charges (see Fig. $4, z=4$ ). Consequently, one of the new vortices annihilates with the primary vortex (Fig. $4, z=5$ ), substituting it by a new vortex of the generated pair. This appears as an overall effect of a large vortex shift (Fig. $4, z=16$ ).

Conclusions. - In this Letter, we have studied an optical analog of the Aharonov-Bohm scattering that allows illustrating, at a macroscopic level, one of the fundamental quantum mechanical phenomena. Moreover, since each specific problem on the geometrical phases has its own peculiarities, in optics we are able to study, for the first time to our knowledge, both weakly and strongly nonlinear effects in the vortex-induced wave scattering characterized by a significant change in the symmetry of the scattered wave and the subsequent stripe instability, unzipping, and break up. Here we have analyzed the NLS model in optics since the experimental verification of the obtained results

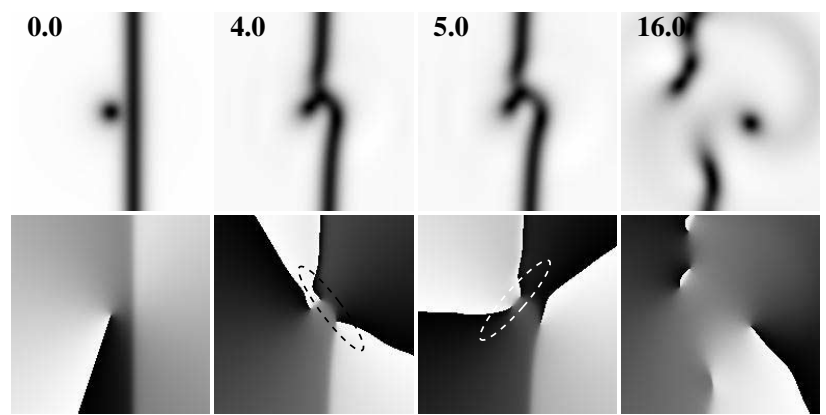

FIG. 4. Vortex pair creation and annihilation during the scattering of the stripe $\left(v^{2}=0.1\right)$, which gives rise to an effectively large vortex shift.

is a subject of our following interest. However, the same model is known to appear in the macroscopic dynamics of the Bose-Einstein condensates, so that our analysis applies to that case as well. We hope our theoretical results can be further verified experimentally, in both nonlinear optics and the physics of ultracold condensed gases.

The work was supported by the Performance and Planning Fund of the Institute of Advanced Studies and by the Australian Photonics Cooperative Research Centre. A. A. N. acknowledges support from the Fund for the Promotion of Research at the Technion and a travel grant of the Israeli-Australian Theeman Foundation. The authors thank M. Berry, D. Pelinovski, L. Pismen, E. B. Sonin, and M. Soskin for their interest in this work and V. Tikhonenko, J. Christou, and B. Luther-Davies for collaboration at the initial stage of this project.

[1] Y. Aharonov and D. Bohm, Phys. Rev. 115, 485 (1959).

[2] M. V. Berry, Proc. R. Soc. London A 392, 45 (1984).

[3] E. B. Sonin, Sov. Phys. JETP 42, 469 (1976); M. V. Berry et al., Eur. J. Phys. 1, 154 (1980).

[4] F. Vivanco et al., Phys. Rev. Lett. 83, 1966 (1999).

[5] M. R. Matthews et al., Phys. Rev. Lett. 83, 2498 (1999); K. W. Madison et al., Phys. Rev. Lett. 84, 806 (2000).

[6] See, e.g., Yu. S. Kivshar and B. Luther-Davies, Phys. Rep. 298, 81 (1998), and references therein.

[7] L. P. Pitaevskii, Sov. Phys. JETP 8, 888 (1959); see also L. M. Pismen, Vortices in Nonlinear Fields (Clarendon, Oxford, 1999), Sec. 4.3.4 and Eq. (4.73).

[8] As a matter of fact, Eq. (3) is valid in the long-wavelength limit when the wave dispersion vanishes, and all linear waves propagate with the same (sound speed) velocity.

[9] Yu. S. Kivshar et al., Opt. Lett. 25, 123 (2000).

[10] See, e.g., Yu. S. Kivshar and D. E. Pelinovsky, Phys. Rep. 331, 117 (2000), and references therein.

[11] Far from the vortex, the numerical results are described by Eq. (12). For example, at $z_{\text {in }}=-16$ and $v^{2}=0.9$, we have $\Delta x(-4)=1.36$, which agrees well with the numerical data, $\Delta x(-4)=1.41 \pm 0.12$. 